\title{
Can We Stop the Academic Al Brain Drain?
}

\author{
Lars Kunze $^{1}$
}

๑) Gesellschaft für Informatik e.V. and Springer-Verlag GmbH Germany, part of Springer Nature 2019

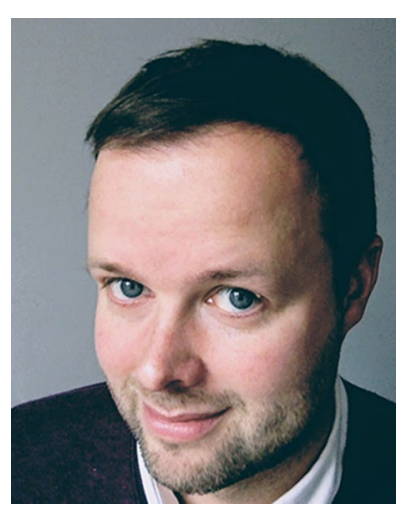

Dear readers,

The AI research landscape is changing. The distinction between academic research and corporate research labs is vanishing. One of the main reasons for this change is the huge success of (deep) machine learning methods over the last five years or so. Another reason is the tremendous advancement in robotic technology in the last decade. These and other factors have brought the realisation and commercialisation of various real-world applications in computer vision, natural language processing and robotics within reach. This has sparked a substantial increase of investment in AI technologies in industry, business and commerce which has led to a high demand of AI researchers in the US, China, and Europe. As a result, more and more of the most talented graduate and $\mathrm{PhD}$ students, postdocs as well as professors and sometimes even whole research teams have left academia to pursue a well-paid career in corporate research.

This academic AI brain drain is not without consequences. On one hand we might see positive effects in industry where AI technologies can be developed faster and deployed in real-world applications from which several groups of people might benefit. However, these AI technologies will only be available to the companies which developed them but not to the research community as a whole. On the other hand, the brain drain will raise profound new questions for the research and education in AI in academia. For example, how can universities attract and retain world-class

Lars Kunze

lars@ robots.ox.ac.uk

1 Department of Engineering Science, Oxford Robotics Institute (ORI), University of Oxford, Oxford, UK researchers? How can they compete with exorbitant salaries offered by tech companies such as Google, Facebook, and Amazon? What are possible incentives for people to stay in academia? Permanent contracts or a dual affiliation model? And who will educate the next generation of AI researchers when the most talented people have left?

The importance of these questions has been widely recognised by governments as well as academic societies and initiatives. In their AI strategy ${ }^{1}$ the Federal Government of Germany sets out the aim to develop existing and establish new centres of excellence for AI to strengthen and safeguard the competitiveness of Germany and Europe in the future. To this end, they plan to offer working conditions and remuneration that are internationally attractive and competitive. In particular, they want to support junior researchers and reinforce academic teaching in AI. The German Informatics Society $(\mathrm{GI})^{2}$ has welcomed this AI strategy in a recent statement. The GI highlighted the importance of consolidating the education in AI at universities as well as in schools. They also stressed that substantial funding for AI research is essential to prevent and/or to reduce an academic AI brain drain. In particular, they pointed out that funding and support should target all areas of AI research and not only those which currently receive most of the attention by the media such as machine learning and data science. Similarly, the CLAIRE initiative ${ }^{3}$, the confederation of laboratories for AI research in Europe, has presented "A European Vision for AI". In their document they describe the need for a European AI strategy to address future challenges such as academic brain drain. Key points of their vision include attracting and fostering academic talent as well as creating a strong infrastructure which can support extensive AI research and compete with large tech companies.

If and how the academic brain drain can be stopped remains an open question. But given the above initiatives we are optimistic that the current trend can be slowed down, and

\footnotetext{
${ }^{1}$ https://www.ki-strategie-deutschland.de.

2 https://gi.de.

3 https://claire-ai.org.
} 
maybe even stopped. Hence, we are convinced that academia will continue to play a key role in AI research in the future. The KI journal will certainly keep you informed about the latest developments.

Lars Kunze

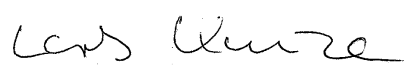

\section{Forthcoming Special Topics}

\subsection{Smart Production}

Guest Editors: Martin Ruskowski (TU Kaiserslautern), Michael Beetz (University of Bremen) and Georg Bartels (University of Bremen).

Smart Production is key for industrial and societal growth, providing opportunities for designing our future work life. This special issue targets to illustrate the requirements and challenges of a smart production which targets not only cyber-physical systems as enabler technology and AIbased robots in production but also solutions for supporting humans in production. For a beneficial use of AI, the necessary infrastructure must be provided. This includes currently used communication protocols and proposed architectures, as well as production systems. Also, contributions concerning the role of $\mathrm{AI}$ in context of robots and human workers, respectively, and industrial use-cases are invited.

Contributions to the following topics are sought:

- Methodological and technological prerequisite for smart production from an AI perspective

- Lean automation as a base concept for self-organizing factory

- Edge computing and architecture

- Intelligent production control/semantic OPC-UA

- Base system for production plants for an efficient variability of a production process

- Current AI applications from research and industry

- Smart HMI

- Advanced analytics and predictive maintenance

- AI-based robotics in production

- Robot assembly/disassembly planning

- Knowledge representation and processing for robots

- Cognition-enabled manipulation

- Robot safety

- Robotic logistics processes

- Perception for grasping and manipulation

- Force and tactile sensing in robot assembly
- Compliant assembly

If you are interested in contributing to this special issue, please contact one of the guest editors.

\subsection{Cognitive Reasoning}

Guest Editors: Ulrich Furbach (University of Koblenz-Landau), Steffen Hölldobler (TU Dresden), Frieder Stolzenburg (Harz University of Applied Sciences).

Human reasoning or the psychology of deduction is well researched in cognitive psychology and in cognitive science. There are many findings which are based on experimental data about human reasoning tasks. Among others, models for the Wason selection task or the suppression task are discussed by psychologists and cognitive scientists. However, only few of these models are computational and often models are modified when applied to a different task.

Automated deduction, on the other hand, mainly focuses on the automated proof search in formal, logical calculi. Indeed, there is tremendous success during the last decades, and automated deduction systems are used in many industrial applications. However, most automated deduction systems are not really concerned with human reasoning tasks. Recently, a coupling of the areas of cognitive science and automated reasoning is addressed in several approaches. For example, there is increasing interest in modeling human reasoning tasks within automated reasoning systems based on answer set programming, deontic logic, abductive logic programming, and various other AI approaches.

This special issue of the Künstliche Intelligenz Journal aims to foster the synergies between cognitive science and automated deduction. The topics of interest for the special issue of the Künstliche Intelligenz Journal include, but are not limited to:

- Limits and differences between automated deduction and human reasoning

- Automated deduction and the psychology of deduction

- Automated deduction and (preferred) mental models

- Common sense reasoning, cognitive science and automated deduction

- Modeling human reasoning tasks using (classical, nonmonotonic or defeasible) logics

- Modeling human reasoning tasks using automated reasoning systems

- Modeling human reasoning tasks using inductive reasoning systems

- Modeling human reasoning tasks using probabilistic reasoning systems

- Applications 
If you are interested in contributing to this special issue, please contact one of the guest editors.

\subsection{Reintegrating Artificial Intelligence and Robotics}

Guest Editors: Federico Pecora (Örebro University), Masoumeh Mansouri (Örebro University), Nick Hawes (University of Oxford), Lars Kunze (University of Oxford).

A major goal of Artificial Intelligence (AI) is to create autonomous, intelligent machines, or robots, that can sense their surroundings, reason about what they have perceived, plan their next actions, and act accordingly to accomplish their tasks. Moreover, robots should be able to learn from their own experience (including interactions with other agents) and adapt to changing conditions within their environments over their lifetime. It is the aim of this special issue to emphasize that the reintegration of AI methods is a nontrivial factor in the design, development and evaluation of robot systems. In particular, we are interested in work related to both fully-integrated robots systems that use methods of AI to perform complex tasks in realistic environments and fundamental AI techniques that have the potential to transform the capabilities of robot systems, but which not been convincingly demonstrated in integrated systems.

Topics of interest include, but are not limited to:

- Knowledge representation and reasoning for robots

- Qualitative representations for robots

- Integrated task and motion planning

- Context-based scene understanding

- Semantic mapping and reasoning with semantic maps

- Constraint-based reasoning for robots

- Continuous planning and on-line problem solving for robots

- AI-enabled human-robot interaction

- Lifelong learning and adaptation

- Verification of autonomous systems

- Reasoning with uncertain and inconsistent knowledge

- System-level AI for robots
If you are interested in contributing to this special issue, please contact one of the guest editors.

\subsection{Artificial Intelligence in Games}

Guest Editors: Sebastian Risi (IT University of Copenhagen) and Mike Preuss (University of Münster).

This special issue focuses on Artificial Intelligence (AI) methods applied in and for different types of games (e.g., board games, video games, serious games). Games have been shown to be the perfect testbed for advanced AI methods. AI in games is now a well established research area with two dedicated conferences and as well as a dedicated journal. Especially deep learning methods have recently proven to beat the best human experts in Atari video games and the game Go. Other methods such as evolutionary computation have been shown to allow complete new types of games through procedural content generation. While there has been much progress in game AI recently, some games such as StarCraft remain beyond even the most advanced AI algorithms. The goal of this special issue is to present a survey of the current research in Game AI and emerging trends in this area.

Some key topics of interest include:

- Procedural content generation

- Games as AI testbeds

- AI for NPCs (non-player characters)

- Player modelling

- Game mining

- Self-play

- Game balancing

- Human-computer interaction

- Human-based computation in games.

If you are interested in contributing to this special issue, please contact one of the guest editors. 